\title{
From Saccharomyces cerevisiae to human: The important gene co-expression modules
}

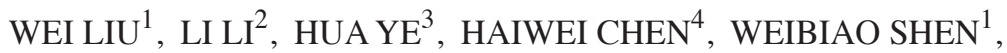 \\ YUEXIAN ZHONG ${ }^{1}$, TIAN TIAN ${ }^{1}$ and HUAQIN HE ${ }^{1}$ \\ ${ }^{1}$ School of Life Sciences, Fujian Agriculture and Forestry University, Fuzhou, Fujian 350002; ${ }^{2}$ Institute of Health \\ Service and Medical Information, Academy of Military Medical Sciences, Beijing 100850; ${ }^{3}$ Department of \\ Gastroenterology, Ningbo Medical Center, Lihuili Hospital, Ningbo, Zhejiang 315040; ${ }^{4}$ Department of \\ Cardiology, First Affiliated Hospital of General Hospital of PLA, Beijing 100048, P.R. China
}

Received April 8, 2017; Accepted May 18, 2017

DOI: $10.3892 /$ br.2017.941

\begin{abstract}
Network-based systems biology has become an important method for analyzing high-throughput gene expression data and gene function mining. Yeast has long been a popular model organism for biomedical research. In the current study, a weighted gene co-expression network analysis algorithm was applied to construct a gene co-expression network in Saccharomyces cerevisiae. Seventeen stable gene co-expression modules were detected from 2,814 S.cerevisiae microarray data. Further characterization of these modules with the Database for Annotation, Visualization and Integrated Discovery tool indicated that these modules were associated with certain biological processes, such as heat response, cell cycle, translational regulation, mitochondrion oxidative phosphorylation, amino acid metabolism and autophagy. Hub genes were also screened by intra-modular connectivity. Finally, the module conservation was evaluated in a human disease microarray dataset. Functional modules were identified in budding yeast, some of which are associated with patient survival. The current study provided a paradigm for single cell microorganisms and potentially other organisms.
\end{abstract}

\section{Introduction}

The budding yeast, Saccharomyces cerevisiae, has been used to make bread and beer for thousands of years. As a single-cell organism, budding yeast has been extensively investigated in genetics and physiology as a model system for eukaryotes, due to its well-annotated genome and short life cycle (1).

Correspondence to: Dr Huaqin He, School of Life Sciences, Fujian Agriculture and Forestry University, No. 15 Shangxiadian Road, Fuzhou, Fujian 350002, P.R. China

E-mail: hehq3@fafu.edu.cn

Key words: annotation, gene co-expression network, Saccharomyces cerevisiae, survival analysis
Completion of the budding yeast genome sequencing project helped to determine a total of 6,275 genes on 16 chromosomes (12 million base pairs). Yeast possesses $23 \%$ homologous genes to humans; therefore, it is considered as a useful model for gene function studies (2). Although yeast and human diverged from a common ancestor $\sim 1$ billion years ago, lines of evidence demonstrate the strong conservation of gene function between yeast and humans (3).

The advancement of cDNA array technology and its low cost make genome-wide gene expression analysis possible. There are many transcriptome data of budding yeast in public databases, including gene expression data from different nutritional conditions, growth stages and gene knock-out models (4). A task for bioinformaticians is to reanalyze these large-scale microarray data and next-generation sequencing data, and identifying the hidden information within these databases. Systems biology studies use high-throughput data and mathematical models to construct yeast transcriptional, gene regulation and protein interaction networks $(5,6)$. The current study used the Google Scholar search engine (https:// scholar.google.com/) and, to the best of our knowledge, weighted gene co-expression network analysis (WGCNA) has not been applied to budding yeast. Using WGCNA to construct a yeast gene co-expression network has many advantages. Firstly, individual experiments may lose meaningful biological information due to relatively small sample sizes and different statistical methods. Pooling datasets from studies helps to strengthen statistical power. Secondly, the gene co-expression analysis reduces high-dimensional genome-wide gene expression to only tens of modules, which simplifies the depiction of yeast biology functions. Thirdly, functionally unknown genes are inferred from co-expressed gene modules by the principle of guilt by association (7). Finally, the current analysis may provide a paradigm for single-cell microorganism.

The aim of the present study was to construct a budding yeast gene co-expression network and identify functional modules that may represent different aspects of yeast biological function. It is hypothesized that certain highly connected genes may be involved in module function. The conservation of these modules is further validated in human tumor cells, 
some of which may differentiate the survival times of separate patients into long and short.

\section{Materials and methods}

Microarray data processing. Yeast microarray data were downloaded from Gene Expression Omnibus (GEO) (http:// ncbi.nlm.nih.gov/gds) (8). Detailed information of the 218 gene expression datasets used in the current study are available at Online Resource 1 (http://bioinformatics.fafu.edu. $\mathrm{cn} /$ Downloads.html). To simplify the data analysis procedure and to enhance reproducibility, only 2,814 budding samples from experiments run on Affymetrix Yeast Genome 2.0 Array (Thermo Fisher Scientific, Inc., Waltham, MA, USA) were included. Datasets from fission yeast was excluded. Expression Console (version 1.2) (Affymetrix, Inc., Santa Clara, CA, USA) was used to process raw data. Probe level expression data were extracted using the MAS5.0 method and normalized using the DNAMR package (http://www.rci.rutgers. edu/ cabrera/DNAMR).

Weighted gene co-expression network construction and module detection. Signed network was constructed according to WGCNA protocols (9-11). The WGCNA R function was implemented using the following parameters: power $=12$, minModuleSize $=30$, deepSplit=4, networkType $=$ 'signed'. Briefly, Pearson correlation coefficients were calculated for all pair-wise comparisons of the probes across all samples. The resulting correlation matrix was converted into a matrix of connection strengths (that is, an adjacency matrix) using the power function $\alpha_{i j}=\left[0.5+0.5 \times \operatorname{cor}\left(x_{i}, x_{j}\right)\right]^{\beta}$ where $x_{i}$ and $x_{j}$ are the $i$ th and $j$ th probe expression, which resulted in a weighted network. Then topological overlap measure (TOM) was calculated as follows:

$$
\mathrm{TOM}=\frac{\sum_{u \neq i, j} a_{i u} a_{u j}+a_{i j}}{\min \left(\mathrm{k}^{\mathrm{k}} \operatorname{total}_{i}, \mathrm{k} \cdot \text { total }_{j}\right)+1-a_{i j}}
$$

Calculation of 1-TOM was performed as a biologically meaningful measure of node similarity, representing how close the neighbors of probe 1 were to the neighbors of probe 2. Probes were hierarchically clustered using 1-TOM as the distance and modules were determined by choosing a height cutoff of 0.995 for the resulting dendrogram. Highly similar modules were identified by clustering and merged together using a dynamic tree-cutting algorithm (7). The module eigengene (ME) corresponds to the first principal component of a given module and is calculated as follows: $\mathrm{ME}=\operatorname{princomp}\left(x_{\mathrm{il}}^{(\mathrm{q})}\right)$, where $\mathrm{i}$ corresponds to module probes and 1 represents microarray samples in module q. This may be considered as the most representative probe expression in a module. Module membership ( $\mathrm{MM}$ or $\mathrm{k}_{\mathrm{ME}}$ ) for each probe in each module refers to the Pearson correlation between the expression level of the probe and ME (7). MM was calculated by $\mathrm{K}_{\text {cor, }}^{(q)}=\operatorname{cor}\left(x_{\mathrm{i}}, E^{(q)}\right)$, where $x_{\mathrm{i}}$ is the profile of node $i$ and $E^{(q)}$ is the ME of module $q$. Hub genes were defined as corresponding probes that have high module membership values within a module (11). The stability of each module was evaluated by sampling 1,407 samples from all 2,814 samples 1,000 times. Correlation between connectivity calculated from the sampled

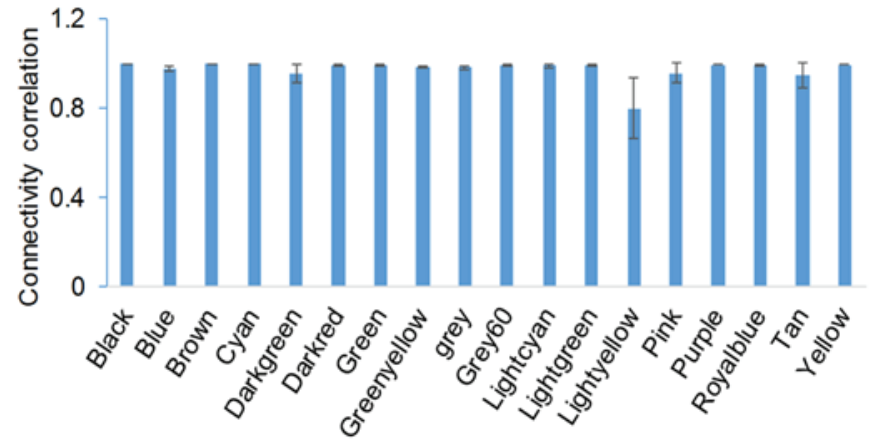

Figure 1. Correlation of intramodule connectivity for each module after sampling 1,000 times (means \pm standard deviation).

samples and the original samples was represented as the mean \pm standard deviation.

Gene ontology (GO) and pathway enrichment analysis. GO enrichment and Kyoto Encyclopedia of Genes and Genomes pathway analysis for network modules were performed using the Database for Annotation, Visualization and Integrated Discovery (DAVID 6.7, https://david-d.ncifcrf.gov) with the background list of all genes on the array (12-14). In DAVID, the overrepresentation of a term is defined as a modified Fisher's exact P-value with an adjustment for multiple tests using the Benjamini-Hochberg method (15).

Survival analysis. A human breast cancer dataset, GSE31448, colorectal cancer dataset, GSE17536, and sarcoma dataset, GSE21050 were downloaded from the NCBI GEO database. Raw files were processed using the Affymetrix Expression Console. The yeast module genes were mapped to human genes using the NCBI HomoloGene system (https://www.ncbi. nlm.nih.gov/homologene). To obtain the module expression value MEs, the three disease gene expression datasets were projected to yeast modules according to homologous genes. Patients were then separated into two groups with high and low MEs. Survival analysis was conducted to compare the survival time difference in $\mathrm{R}$ using the survival package. P-values for survival curves were determined from the Kaplan-Meier survival curves by use of the log-rank test.

\section{Results}

WGCNA. A large budding yeast gene co-expression network was constructed in the present study, to the best of our knowledge, for the first time. WGCNA identified 17 modules, which contained genes with similar expression patterns. To evaluate the stability of modules, gene connectivity was correlated before and after sampling (Fig. 1). Module stability was expressed as the correlation of intramodule connectivity between the original and sampled module. The average correlation was $>0.8$. Module Cyan was the most stable module, and module Lightyellow was the least stable (Fig. 1). All gene modules and corresponding connectivity data are provided in Online Resource 2 (http:// bioinformatics.fafu.edu.cn/Downloads.html).

Modules are involved in different functions. To identify modular features, DAVID was used to characterize module 
Table I. GO and KEGG annotation of the identified 17 gene co-expression modules in Saccharomyces cerevisiae.

GO term (Benjamini-Hochberg adjusted P-value)

Module

(no. genes)

Black (277) Response to heat (1E-45)

Blue (935)

Brown (796)

Cyan (111)

Dark green (37)

Dark red (49)

Green (736)

Green yellow

(135)

Grey 60 (84)

Light cyan (85)

Light green

(187)

Light yellow

(57)

Pink (194)

Purple (143)

Royal blue (52) Sulfur metabolism (2E-25)

$\operatorname{Tan}(1851)$

Yellow (666)

Cell cycle phase (8E-10)

Regulation of translation (4E-27)

Oxidative phosphorylation (2E-38)

$$
M \text { phase of meiotic }
$$
cell cycle (2E-22)

Amino acid

biosynthesis (2E-23)

Autophagy (1E-19)

Mitochondrial translation (7E-98)

Steroid metabolism (1E-5)

Ubiquitin-dependent

$$
\begin{gathered}
\text { Protein amino acid } \\
\text { glycosylation (2E-13) } \\
\text { Oligosaccharide } \\
\text { metabolic process (3E-4) } \\
\text { Sporulation (5E-4) } \\
\text { Organic acid }
\end{gathered}
$$
catabolic process (4E-11)

Monosaccharide catabolic process (3E-2) protein metabolism (1E-38)

Intrinsic to membrane (4E-34)

Condensed nuclear chromosome (3E-10)

Glycine cleavage complex (7E-3)

\section{Vacuole (5E-4)}

Mitochondrial ribosome (7-E101)

Intrinsic to membrane (5E-7)

Proteasome (2E-58)

ncRNA processing (1E-100)
Cytosolic ribosome (2E-10)

Nucleolus (2E-128)
KEGG

(Benjamini-Hochberg adjusted P-value)

Sucrose metabolism (7E-7)

Cell cycle (4E-12)

Ribosome (5E-66)

Oxidative phosphorylation (4E-31)

Meiosis (7E-7)

Structure-specific

DNA binding (1E-04)

Oxidoreductase activity, acting on the $\mathrm{CH}-\mathrm{NH} 2$ group of donors, disulfide as acceptor (3E-2)

Transcription regulator activity (9E-4)

Structural constituent of ribosome (8E-69)

O-acyltransferase activity (1E-3)

Threonine-type endopeptidase activity (3E-21)

Mannosyltransferase activity(1E-3)

Aminoacyl-tRNA biosynthesis (8E-7)

Proteasome (2E-50)

N-Glycan biosynthesis (5E-9)

Ligase activity, forming carbon-sulfur bonds (2E-2)

Sulfur amino acid transmembrane transporter activity (6E-5)

Structural constituent of ribosome (9E-5)

DNA-directed RNA polymerase activity (2E-14)
Arginine and proline metabolism (3E-3)

\section{Glyoxylate and dicarboxylate metabolism (5E-6) Sulfur metabolism (5E-10) \\ Ribosome (3E-10) \\ Pyrimidine} metabolism (9E-16)

GO, gene ontology; KEGG, Kyoto Encyclopedia of Genes and Genomes.

genes (Table I). All the modules were associated with distinct biological functions, representing aspects of yeast cell function. Module yellow is enriched with genes associated with ncRNA processing, which predominantly localize in the nucleolus. Both modules cyan and green-yellow were associated with mitochondria, while module cyan encodes proteins involved with oxidative phosphorylation on mitochondrial membranes, and module green-yellow encodes mitochondrial ribosome proteins.

Functions of module hub genes. In a network biology, genes do not contribute equally to a module. Those genes with higher connectivity may exert more significant roles in a module. To establish the functional annotation of those genes, the top 
Table II. Hub genes and their encoding proteins of the gene co-expression modules in Saccharomyces cerevisiae.

\begin{tabular}{lll}
\hline Module & \multicolumn{1}{c}{ Gene } & \\
\hline Black & DCS2 & Encoding protein \\
Blue & YDR506C & Protein DCS2 \\
Brown & PPL22A & Putative multicopper oxidase YDR506C \\
Cyan & SDH2 & S0S ribosomal protein L22-A \\
Dark green & IME2 & Succinate dehydrogenase [ubiquinone] iron-sulfur subunit, mitochondrial \\
Dark red & ARG7 & Meiosis induction protein kinase IME2/SME1 \\
Green & GPX1 & Arginine biosynthesis bifunctional protein ARG7, mitochondrial \\
Green yellow & MRPL49 & Glutathione peroxidase 1 \\
Grey 60 & PAU5 & 54S ribosomal protein L49, mitochondrial \\
Light cyan & RPT4 & Seripauperin-5; Seripauperin-7 \\
Light green & ALG8 & 26S protease subunit RPT4 \\
Light yellow & PRP39 & Dolichyl pyrophosphate Glc1Man9GlcNAc2 alpha-1,3-glucosyltransferase \\
Pink & MER1 & Pre-mRNA-processing factor 39 \\
Purple & FOX2 & Meiotic recombination 1 protein \\
Royal blue & MET16 & Peroxisomal hydratase-dehydrogenase-epimerase \\
Tan & SPBC947.09 & Phosphoadenosinephosphosulfate reductase \\
Yellow & RPF2 & Uncharacterized protein C947.09 \\
& & Ribosome biogenesis protein RPF2
\end{tabular}

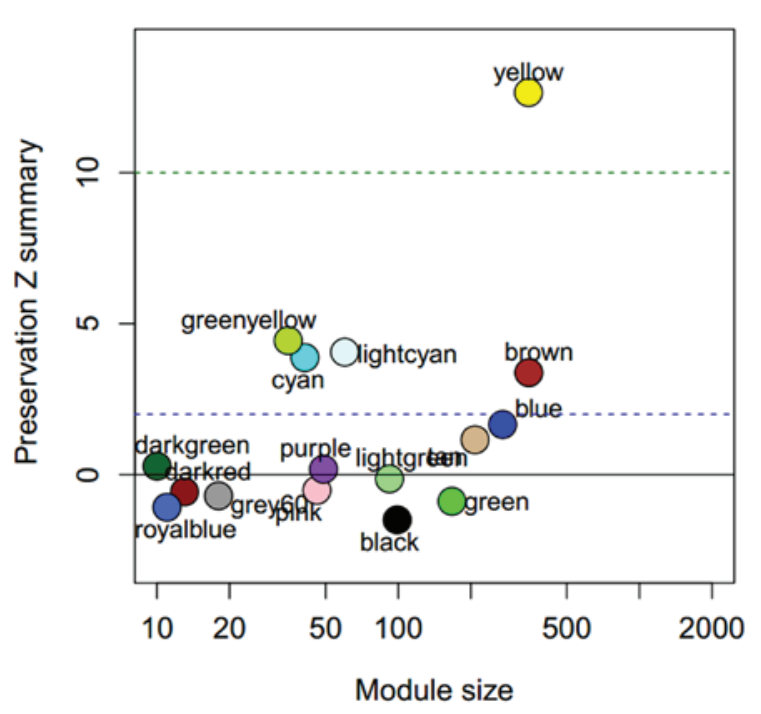

Figure 2. Module preservation analysis between bake yeast and human cancer cell lines. Z summary represents the summary preservation statistics, where the $\mathrm{y}$-axis demonstrates the preservation statistics and the $\mathrm{x}$-axis is the gene numbers in each module. The dashed blue and green lines indicate the thresholds $Z=2$ and $Z=10$, respectively. $Z$ summary $<2$ implies no evidence for module preservation, $\mathrm{Z}$ summary $\leq 10$ indicates weak to moderate evidence, while $\mathrm{Z}$ summary $>10$ suggests strong evidence for module preservation. The statistics are derived from 1,000 permutations.

hub genes were screened to examine their functions (16). For example, module blue was enriched in cell cycle-associated genes. The top hub gene of the module was YDR506C, which contributes to nuclear division and genome integrity $(17,18)$. The results are presented in Table II.

Module conservation in human cancer cell lines. WGCNA ModulePreservation function was used to evaluate the preservation of the 17 yeast modules in the human cancer cell lines dataset, GSE36133 (Fig. 2). Five modules, yellow,

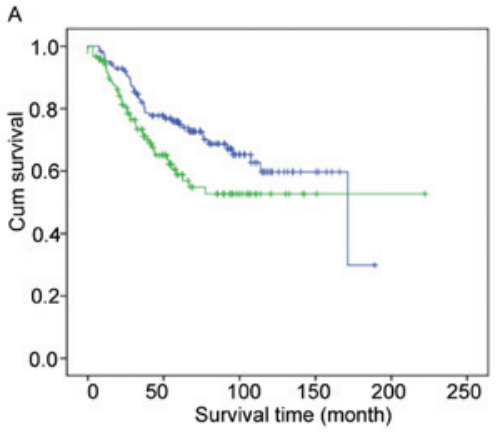

B
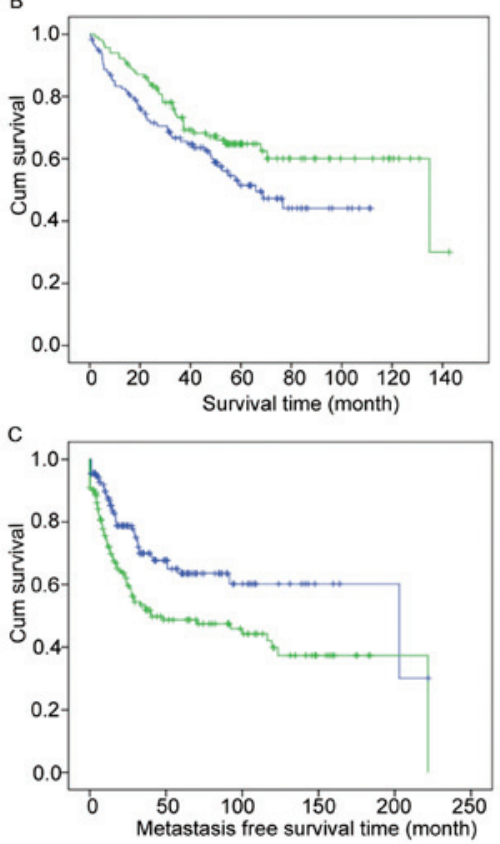

Figure 3. Identified modules differentiate between patients with different survival/metastasis-free survival time. (A) Breast cancer patients (dataset, GSE31448). (B) Colorectal cancer patients (dataset, GSE17536). (C) Sarcoma patients (dataset, GSE21050). P-values were computed using Log-rank test. Blue line, low ME group; green line, high ME group. ME, module eigengene. 
green-yellow, light cyan, cyan and brown were identified to be well preserved from high to low in human cancer cell lines. They are associated with ncRNA processing, mitochondrial translation, ubiquitin-dependent protein metabolism, oxidative phosphorylation and regulation of translation.

Co-expression modules differentiate between patients with different survival times. To further validate the importance of these modules, human breast cancer, colorectal cancer and sarcoma microarray datasets were used to plot survival curves. For example, module blue differentiates between breast cancer patients (Fig. 3A); module royal blue separates colorectal cancer patients (Fig. 3B); and module light green distinguishes sarcoma patients (Fig. 3C).

\section{Discussion}

WGCNA has been extensively applied to gene co-expression network construction and analysis in various species. For example, in human brain transcriptome analysis, Oldham et al (10) identified the gene co-expression corresponding to different brain regions. In plants, Zhan et al (19) identified cell-type specific gene co-expression modules, and observed regulatory modules that were associated with endosperm cell differentiation. WGCNA was previously used to depict functional gene co-expression modules in mouse liver and human cancer cell lines $(20,21)$. In the present study, a gene network of budding yeast was successfully constructed using WGCNA analysis. All of the identified 17 modules were associated with specific functional categories. As a single cell organism, the results are easier to interpret. Therefore, WGCNA has an advantage over differential gene expression analysis or ANOVA, which compare two or more experimental groups. When there are many different biological groups, it is more complicated to analyze these data. WGCNA surmount these disadvantages, as it simplifies thousands of genes into tens of functional modules. Finally, the method does not require prior knowledge, so novel gene functions may be identified. WGCNA has previously been used as a gene annotation method (22).

The 17 identified modules represent different aspects of budding yeast functions, including substance and energy metabolism, cell proliferation and stimulus response (Table I). Module black contains genes associated with heat response, which is an important trait of yeast function (23). Recent studies indicate that yeast has an adaptation for environmental stress, such as high temperature (24). Substance metabolism modules, include amino acid metabolism (dark red), steroid metabolism (grey 60), organic acid metabolism (purple) and sulfur metabolism (royal blue). Each module has a distinct function, indicating the robustness of WGCNA.

Only 1,944 module genes were projected to human homologous genes due to the limited number of yeast genes on microarray. Thus, there is no definitive conclusion that modules with a low preservation $\mathrm{Z}$ summary value are not preserved within humans as a result of fewer genes in these modules (Fig. 2). However, the five preserved modules identified in the present study are consistent with a previous study that demonstrated that genes within these modules are replaceable (3).
The significance of cancer cell line gene co-expression modules in tumors has previously been reported (21). In the current study, yeast modules were observed in various human cancer datasets. For example, certain modules differentiate between patients with long and short survival times, indicating their importance from yeast to humans. Those modules may be crucial in cancer biology and provide information for human tumor research within yeast cells.

\section{Acknowledgements}

The present study was supported in part by the National Natural Science Foundation of China (grant nos. 31270454 and 81502091), and of Zhejiang Provincial Natural Science Foundation (grant no. LQ14H030001) and a Ningbo Natural Science Foundation Grant (grant no. 2013A610232).

\section{References}

1. Botstein D, Chervitz SA and Cherry JM: Yeast as a model organism. Science 277: 1259-1260, 1997.

2. Jiang Q, Lin L and Wang T: A new model for apoptosis research: Yeast. Prog Biochem Biophys 35: 361, 2008.

3. Kachroo AH, Laurent JM, Yellman CM, Meyer AG, Wilke CO and Marcotte EM: Evolution. Systematic humanization of yeast genes reveals conserved functions and genetic modularity. Science 348: 921-925, 2015.

4. Barrett T, Troup DB, Wilhite SE, Ledoux P, Rudnev D, Evangelista C, Kim IF, Soboleva A, Tomashevsky M and Edgar R: NCBI GEO: Mining tens of millions of expression profiles - database and tools update. Nucleic Acids Res 35 (Database): D760-D765, 2007.

5. Zhu J, Zhang B, Smith EN, Drees B, Brem RB, Kruglyak L, Bumgarner RE and Schadt EE: Integrating large-scale functional genomic data to dissect the complexity of yeast regulatory networks. Nat Genet 40: 854-861, 2008.

6. Guelzim N, Bottani S, Bourgine P and Képès F: Topological and causal structure of the yeast transcriptional regulatory network. Nat Genet 31: 60-63, 2002.

7. Zhang B and Horvath S: A general framework for weighted gene co-expression network analysis. Stat Appl Genet Mol Biol 4: Article17, 2005.

8. Barrett T, Troup DB, Wilhite SE, Ledoux P, Evangelista C, Kim IF, Tomashevsky M, Marshall KA, Phillippy KH, Sherman PM, et al: NCBI GEO: Archive for functional genomics data sets - 10 years on. Nucleic Acids Res 39 (Database): D1005-D1010, 2011.

9. Miller JA, Horvath S and Geschwind DH: Divergence of human and mouse brain transcriptome highlights Alzheimer disease pathways. Proc Natl Acad Sci USA 107: 12698-12703, 2010.

10. Oldham MC, Konopka G, Iwamoto K, Langfelder P, Kato T, Horvath S and Geschwind DH: Functional organization of the transcriptome in human brain. Nat Neurosci 11: 1271-1282, 2008.

11. Langfelder P and Horvath S: WGCNA: An R package for weighted correlation network analysis. BMC Bioinformatics 9: $559,2008$.

12. Huang W, Sherman BT and Lempicki RA: Systematic and integrative analysis of large gene lists using DAVID bioinformatics resources. Nat Protoc 4: 44-57, 2009.

13. Huang W, Sherman BT and Lempicki RA: Bioinformatics enrichment tools: Paths toward the comprehensive functional analysis of large gene lists. Nucleic Acids Res 37: 1-13, 2009.

14. Ashburner M, Ball CA, Blake JA, Botstein D, Butler H, Cherry JM, Davis AP, Dolinski K, Dwight SS, Eppig JT, et al: The Gene Ontology Consortium: Gene ontology: Tool for the unification of biology. Nat Genet 25: 25-29, 2000.

15. Benjamini Y and Hochberg Y: Controlling the false discovery rate: A practical and powerful approach to multiple testing. J R Stat Soc B 57: 289-300, 1995.

16. Langfelder P, Mischel PS and Horvath S: When is hub gene selection better than standard meta-analysis? PLoS One 8: e61505, 2013. 
17. Gallina I, Colding C, Henriksen P, Beli P, Nakamura K, Offman J, Mathiasen DP, Silva S, Hoffmann E, Groth A, et al: Cmr1/WDR76 defines a nuclear genotoxic stress body linking genome integrity and protein quality control. Nat Commun 6: $6533,2015$.

18. Brar GA, Yassour M, Friedman N, Regev A, Ingolia NT and Weissman JS: High-resolution view of the yeast meiotic program revealed by ribosome profiling. Science 335: 552-557, 2012.

19. Zhan J, Thakare D, Ma C, Lloyd A, Nixon NM, Arakaki AM, Burnett WJ, Logan KO, Wang D, Wang X, et al: RNA sequencing of laser-capture microdissected compartments of the maize kernel identifies regulatory modules associated with endosperm cell differentiation. Plant Cell 27: 513-531, 2015.

20. Liu W and Ye H: Co-expression network analysis identifies transcriptional modules in the mouse liver. Mol Genet Genomics 289 847-853, 2014
21. Liu W, Li L and Li W: Gene co-expression analysis identifies common modules related to prognosis and drug resistance in cancer cell lines. Int J Cancer 135: 2795-2803, 2014.

22. Childs KL, Davidson RM and Buell CR: Gene coexpression network analysis as a source of functional annotation for rice genes. PLoS One 6: e22196, 2011.

23. Novick $P$ and Botstein D: Phenotypic analysis of temperature-sensitive yeast actin mutants. Cell 40: 405-416, 1985.

24. Li C, Qian W, Maclean CJ and Zhang J: The fitness landscape of a tRNA gene. Science 352: 837-840, 2016. 